each group. EACA treatment resulted in an eightfold increase in the incidence of deepvein thrombosis, but treatment did not affect the incidence of pulmonary embolism. Overall, $78 \%$ of patients who were rated as Hunt-Hess grades 1-3 in the EACA-treated group had a 3-month favorable outcome compared with $65 \%$ of such patients in the non-EACA group; for patients who were rated as grades 4 or 5 , the respective proportions were $22 \%$ and $15 \%$.

The authors conclude that acute antifibrinolytic therapy in patients with aneurysmal subarachnoid hemorrhage should be assessed in a randomized placebo-controlled trial to determine whether this treatment should become the standard of care.

Original article Starke RM et al. (2008) Impact of a protocol for acute antifibrinolytic therapy on aneurysm rebleeding after subarachnoid hemorrhage. Stroke 9: 2617-2621

\section{Stimulation of the subthalamic nucleus targets specific motor symptoms in Parkinson disease}

Deep brain stimulation (DBS) of the subthalamic nucleus has been shown to improve motor symptoms associated with Parkinson disease (PD), but the mechanism of this action is unclear. Previous studies have demonstrated that DBS of the subthalamic nucleus alters regional cerebral blood flow (rCBF) throughout the brain. In a new study, Karimi et al. investigated whether changes in blood flow in specific regions of the brain leads to improvements in different motor functions in PD.

Data were assessed from 31 patients with PD (average age 61 \pm 10 years, average duration since diagnosis $14 \pm 8$ years) who underwent PET scans to measure rCBF responses to bilateral DBS of the subthalamic nucleus. The scans revealed that, after DBS, rCBF was increased in the bilateral thalami and right midbrain and decreased in the right premotor cortex. Improvement in motor symptoms occurred in all patients after administration of DBS. Rigidity improved by $62 \%$ and bradykinesia by $44 \%$, and these effects correlated with decreased rCBF in the supplementary motor area, and increased rCBF in the thalamus, respectively. Postural stability improved by $56 \%$, an effect that was correlated with decreased rCBF in the pedunculopontine nucleus region.

The authors suggest that the relationship between changes in rCBF in specific regions of the brain and improvements in selected motor functions supports the use of targeted treatments in PD.

Original article Karimi M et al. (2008) Subthalamic nucleus stimulation-induced regional blood flow responses correlate with improvement of motor signs in Parkinson disease. Brain [doi:10.1093/brain/awn 179]

\section{Generation of motor neurons from skin fibroblasts of a patient with ALS}

US researchers have generated induced pluripotent stem (iPS) cells from the skin fibroblasts of an 82-year-old woman with amyotrophic lateral sclerosis (ALS). In addition, the investigators successfully directed the iPS cells to differentiate into motor neurons and glial cells. The findings raise the possibility that patientspecific regenerative therapy for motor neuron diseases could be feasible.

Dimos et al. took skin biopsy samples from a woman with a familial form of ALS. The woman was heterozygous for the Leu144Phe allele of the SOD1 (superoxide dismutase) gene, which is associated with a slowly progressing form of ALS. Fibroblasts from the samples were transfected with VSVg pseudotyped retroviruses to introduce the four genes that have previously been shown to reprogram human fibroblasts to a pluripotent state: KLF4, SOX2, OCT4 and c-MYC. After 2 weeks, a small number of transformed cells had given rise to cells morphologically similar to embryonic stem cells. These cell lines were genetically identical to the parent fibroblasts, except for the four viral transgenes with which they had been transduced, and had a gene-expression profile similar to that of pluripotent cells. The iPS cells readily formed embryoid bodies in suspension; these bodies, when subjected to a directed differentiation protocol, generated motor neurons and glial cells.

The generation of patient-specific iPS cells provides an important new method for studying the pathogenesis of both familial and sporadic ALS. Use of the cells for regenerative therapy, however, must await more-controlled 